\title{
A Distance Metric Computation Model for the Design and Development of Palm Vein Recognition System
}

\author{
Shriram D. Raut ${ }^{1}$ and Dr. Vikas T. Humbe ${ }^{2}$ \\ ${ }^{l}$ School of Computational Sciences, Solapur University, Solapur, Maharashtra, \\ India \\ ${ }^{2}$ School of Technology, S. R. T. M. U. N., Sub-Centre, Latur, Maharashtra, India \\ ${ }^{1}$ profraut@ymail.com, ${ }^{2}$ vikashumbe@gmail.com
}

\begin{abstract}
Palm vein recognition is a part of physiological biometric uses blood vessel structure for the identification. The blood transfusion from body parts to heart is made by the blood vein. The blood vein carries de-oxygenated hemoglobin that can be sensed under near infrared light. The pattern classification proposes a design cycle to build a system for recognition. The image database was obtained from Hong Kong Polytechnic University. The experimentation was carried on palm vein image captured under near infrared light. The distance metric computation was done and stored in the form of the template. The paper discuss about the experimentation of Euclidean distance metric on the palm vein feature extracted binary image using Gabor filter and canny edge detection.
\end{abstract}

Keywords- Palm vein, pattern recognition, near infrared, Gabor, canny, Euclidean

\section{Introduction}

Biometric is a leading aspects of person identification using physiological and behavioral characteristics [1]. The physiological traits are mainly face, palm print, iris, palm vein, and finger etc. as the external characteristic [2] of human being. The traits such as keystroke, signature, gait and voice etc. are the behavioral characteristics [3, 4]. The vein biometrics is characterized on the basis of blood vascular structure. The researcher has studied extensively and differentiated palm vein biometrics from other traits, while considering the cost, performance, user convenience, system security and inters operability. The multispectral palm vein image database [5] consists of the image captured under blue, green, and red, near infrared light illumination; in order to acquire the blood vessel structure.

The blood vessel is of two types as artery and vein in which oxygenated and deoxygenated blood flows respectively [6]. 


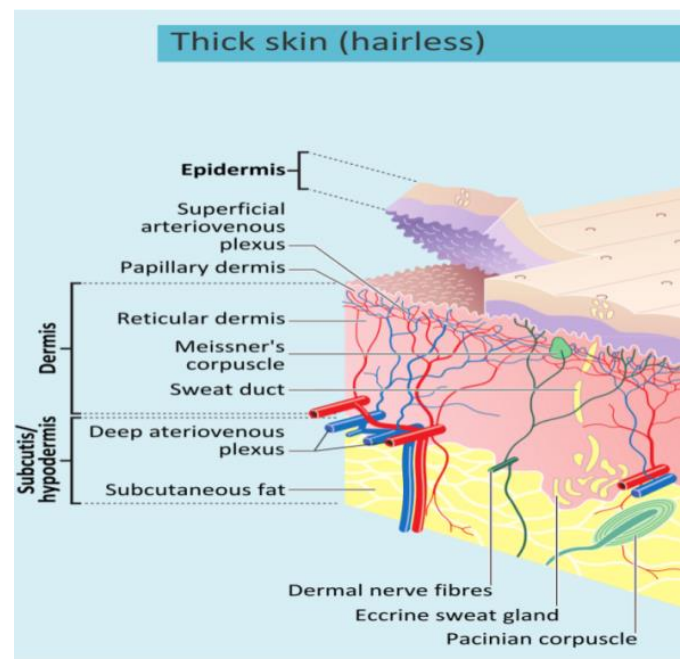

Figure 1. Cross Sectional View of the Skin [Wikipedia]

The deoxygenated hemoglobin absorbs the infrared light at $860 \mathrm{~nm} \mathrm{[4,7]} \mathrm{and} \mathrm{gives} \mathrm{a}$ lighter shade of blood vein structure. The pattern recognition is the science of identification and verification of various subject based on the uniqueness of characteristic that the subject has. The system consists of various phases such as data collection, feature extraction and finally the recognition. The paper is based on derivation of existing distance metrics for forming a kind of feature vector. For our study the Euclidean distance has been implemented to compose a feature vector out of extracted feature set. The paper also discuss about the use of Gabor filter and canny edge detection method to extract the blood vein structure.

\section{Literature Review}

Shi Zhao, Yiding Wang, Yunhong Wang [8] paper specifies the extraction of the feature vector. In paper they have proposed a biometric technique over hand-dorsa for the extracting vein structures. Huan Zhang, Dewen Hu [9], paper discusses about an image preprocessing and feature extraction of palm vein image. A paper states that a recognition system can be designed using blood vessel patterns as a personal identification factor. Madhumita Kathuria [10], proposed a Vein Based Personal Identification System (VBPIS), which overcomes the deficiencies usually found in commercially available biometric systems. A paper has proposed a system that uses pattern recognition, image processing technique for extracting and manipulating the vein patterns. Daniel Hartung, Martin Aastrup Olsen [10], this paper is an approach to extract vein minutiae and to transform them into a fixed-length, translation and scale invariant representation where rotations could be easily compensated. The proposed solution based on spectral minutiae was evaluated against other comparison strategies on three different datasets of wrist and palm vein samples. Xiangping Zeng and Weidong Jin [11], introduced the principles and workflows of vein recognition. At the same time, algorithms of vein recognition were researched. This paper focuses on vein image preprocessing, relative algorithms and a series of simulation results of vein image processing.

\section{About Image Database}

The biometric research centre of Hong Kong Polytechnic University has developed multispectral palm print image database [4]. The image of palm print were acquired under red, blue, and green, near infrared light illumination at $660 \mathrm{~nm}, 470 \mathrm{~nm}, 525 \mathrm{~nm}$ and $880 \mathrm{~nm}$ respectively. The image database consists of 6000 images from 500 left and 
right palms. The database of 195 males and 55 females were registered with their palm print in two sessions. The region of interest from captured image was formed by researcher. The database was enrolled with 250 subjects having 12 image of one hand of every color band as red, green and blue, near infrared. The region of interest is square image as shown in fig 2 was extracted. The square image matrix is extracted which has $128 \times 128$ dimension. For the experimentation sample of 100 image of different subject was studied.

An obtained database contains the images captured under red, green and blue light illumination of different subject but as per the literature reviewed $[5,6]$, the image captured under near infrared light illumination gives better outline of the vein pattern as compared to image captured under green, red and blue light illuminations. In our work, the image captured under near infrared light is used for the purpose of experimentation. An image is a square image centered to a palm region of hand and contains palm print features such as principal lines, secondary lines, ridges, wrinkles and blood vessel lines too. The image preprocessing over the database is observed using fundamental digital image processing operation such as sharpening and smoothening. The concept is to study, observe and implement the algorithm s for feature extraction, detection and recognition of person based on palm vein biometrics from the obtained image database.

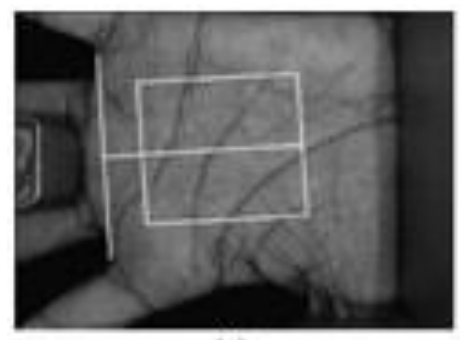

(a)

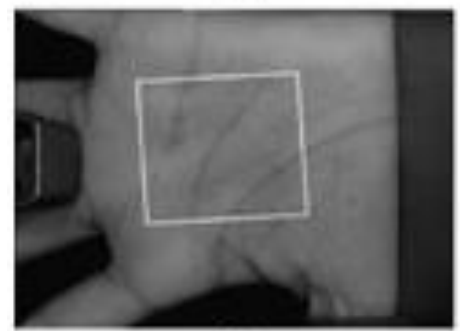

(c)

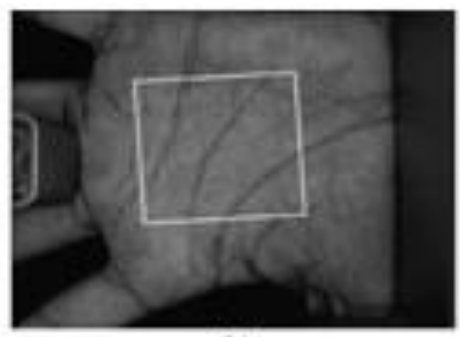

(b)

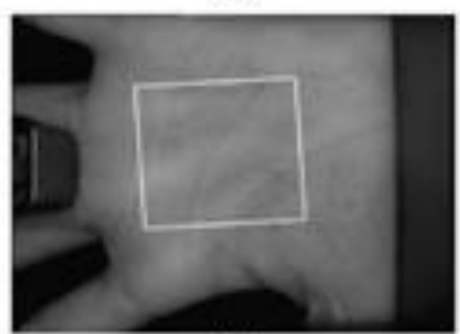

(d)

Figure 1. White Square Marks for the Region of Interest as (a) Blue, (b) Green, (c) Red, (d) NIR

\section{Methodology}

The scientific research approach is adopted for the purpose of experimentation. The pattern recognition design cycle [11] is studied and followed to develop the experimentation.

The steps such as data collection and processing feature extraction followed by classification and decision making. The experimentation is focused on data processing and feature vector set formation. 


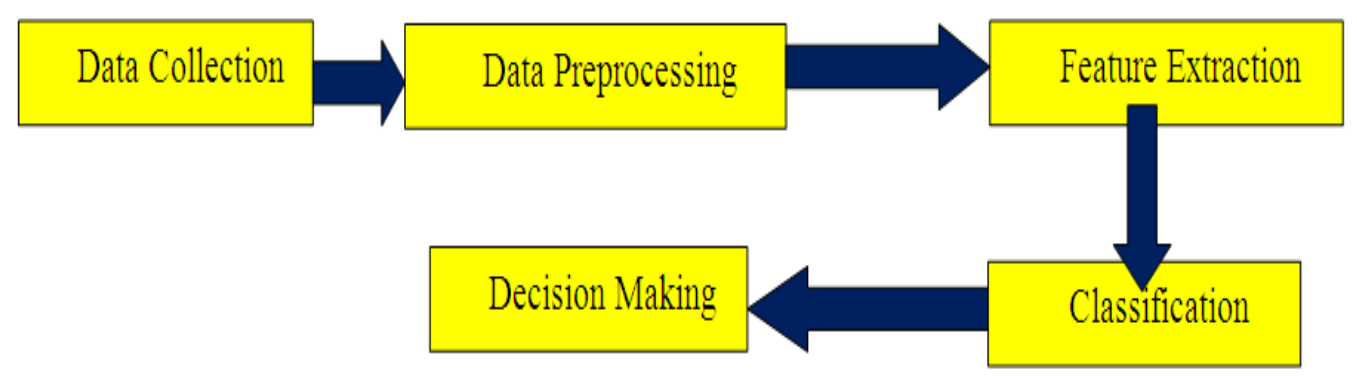

Figure 2. Pattern Recognition Design Cycle

The Gabor filter is used to process the image matrices and extract the blood vessel structure. And further filtered image processed under the canny edge detection algorithm. The Euclidean distance metric of the processed image under Gabor filter and canny edge detector [13] is computed.

A Euclidean distance matrix is a form of distance-square $x_{i j}$ between points taken by pair from a list of points $\left\{x_{\ell}, \ell=1 \ldots N\right\}$; the squared metric, the measure of distancesquare:

$$
x_{i j}=\left|x_{i-} x_{j}\right|^{2} \triangleq<x_{i}-x_{j}, x_{i}-x_{j}>
$$

Considering image of having an m-by-n data matrix $X$, which is treated as $\mathrm{m}$ (1-by-n) row vectors $x_{1}, x_{2}, x_{m}$, the various distances between the vector $x_{s}$ and $x_{t}$ are defined as follows- Euclidean distance

$$
d_{s t}^{2}=\left(x_{s}-x_{t}\right)\left(x_{s}-x_{t}\right)^{\prime}
$$

As per the digital image processing, the Euclidean distance computes the pair wise distance between pairs of pixel objects. The computed distance is stored in the form of feature vector set. The binary vector from the set is to be extracted and further will lead to classify the sample set of one subject with another.

\section{Experiments And Results}

The experimentation is focused to extract the feature vector set from the image sampled to lead a robust recognition system. The results will lead to generate a binary vector form out of Euclidean distance image matrix. The algorithm design is as follows-

i. Read a palm vein image from the image database.

ii. Accept the threshold value for the parameter such as aspect ratio, phase angle, orientation, bandwidth and wavelength.

iii. Now with accepted parameter, apply the Gabor filter.

iv. Perform the canny edge detector [13] on the image as a resultant of the Gabor filter. These operations are useful to extract edges of the blood vein.

v. Compute the Euclidean distance metric on the edge detected image matrix.

vi. Compute the binary vector from the image resulted of Euclidean distance metric. 


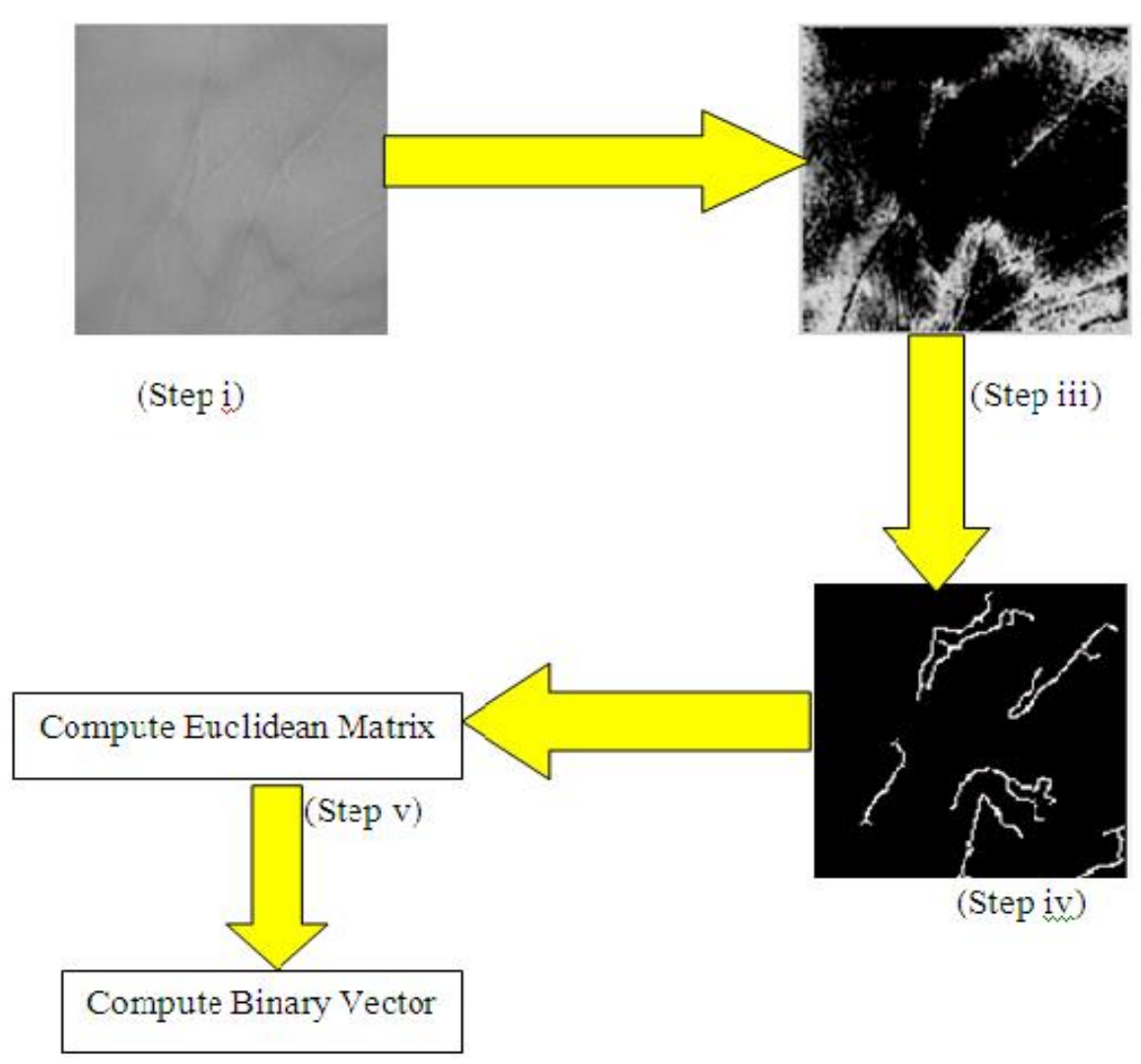

Figure 4. Stepwise Flow of Experimentation

The computed binary vector is to be stored in the form of template as train or enrolled sample. The stated experimentation is a part of the enrollment phase of the recognition system and it can be part of the identification phase too.

\section{Conclusion}

Palm vein biometric is a leading trend to recognize a person based on the uniqueness in the blood vessel structure. The blood vein pattern lies under the skin makes very difficult to forge. The image distance was obtained by the Hong Kong Polytechnic University and has enrolled with 250 subjects and from that experimentation was performed on the 100 image sample of different subjects. The experiment was targeted to extract the edges of the blood vessel and form the feature set of binary vector the Euclidean distance computed matrix. Using this computation the enrollment and identification part of the pattern recognition system can be designed.

\section{Acknowledgments}

We express our gratefulness to Department of Computer Science, Solapur University, Solapur and S. R. T. M. University, Nanded for providing infrastructure and computer laboratory facility to carry rigorous experimentation. We are also thankful to Biometric Research Centre at The Hong Kong Polytechnic University for providing an access of the Multispectral Palm print image database for the purpose of research and development. 


\section{References}

[1] S. Z. Li, "Encyclopedia of Biometrics", New York: Springer, (2009).

[2] A. K. Jain, A. Ross and S. Pankanti, "Biometrics: A Tool for Information Security", IEEE Transactions on Information Forensics and Security, (2006), pp. 125-143.

[3] J. R. Vacca, "Biometric Technologies and Verification Systems", Elsevier, (2007).

[4] S. D. Raut and V. T. Humbe, "Review of Biometrics: Palm Vein Recognition Technique", IBMRD's Journal of Management \& Research, vol. 3, no. 1, (2014), pp. 217-223.

[5] D. Zhang, Z. Guo, G. Lu, L. Zhang and W. Zuo, "An Online System of Multispectral Palm print Verification", IEEE Transactions On Instrumentation And Measurement, vol. 59, no. 02, (2010), pp. 480-490.

[6] P. Zharov, S. Ferguson, J. F. Eidt, P. C. Howard, L. M. Fink and M. Waner, "Infrared Imaging of Subcutaneous Veins", Lasers Surgery Medicine, vol. 34, no. 1, (2010), pp. 56-61.

[7] Y. Zhou and K. Ajay, "Human Identification Using Palm Vein Images", IEEE transactions on information forensics and security, vol. 6, no. 4, (2011).

[8] Z. Xiangping and J. Weidong, "Research of Hand Vein Patterns Recognition for Biometric Identification", IEEE Conference on Biomedical Engineering and Biotechnology, IEEE Computer Society, (2012) pp 884-887.

[9] S. Zhao, Y. Wang and Y. Wang, "Extracting Hand Vein Patterns from Low-Quality Images: A New Biometric Technique Using Low-Cost Devices", Fourth International Conference on Image and Graphics, IEEE Computer Society, (2007), pp. 667-971.

[10] H. Zhang and D. Hu, "A Palm Vein Recognition System", International Conference on Intelligent Computation Technology and Automation, IEEE Computer Society, vol. 1, (2010), pp. 285-288.

[11] M. Kathuria, "Design of a Vein Based Personal Identification System", International Conference on Advances in Recent Technologies in Communication and Computing, IEEE Computer Society, (2010), pp. 284-286.

[12] R. O. Duda, P. E. Hart and D. G. Stock, "Pattern Classification", Wiley Inter Science, Second Ed., (2001).

[13] S. D. Raut and V. T. Humbe, "An Approach to Boundary Extraction of Palm Lines and Vein Pattern", International Journal of Image, Graphics and Signal Processing, vol. 6, issue 12, (2014), pp. 47-52.

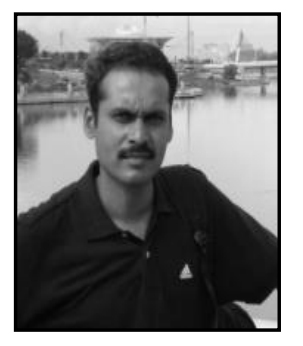

Vikas T. Humbe, has completed his Ph.D. degree from University Department of Computer Science and Information Technology, Dr.B.A.M.U. Aurangabad and working as the Assistant Professor at Department of Computer Science, School of Technology, S.R.T.M. University, Nanded, Sub-campus, Latur. He is a reviewer for various International and National Journals and Conferences like Elsevier's Pattern Recognition Letters, Journal on Machine Vision and Applications, Academic Journals, IEEE IJCNN 07 and 09, ACVIT-09 etc. He is Member of IACSIT Singapore, Member of IAEng, Hong Kong, CSTA, USA and Graduate Member of IEEE-USA. He is the IEEE Graduate Member and has immense research recognition worldwide.

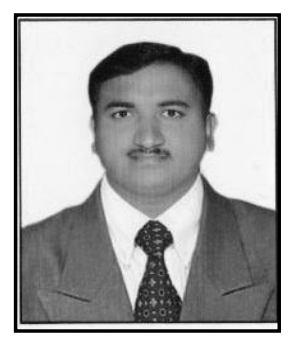

Shriram D. Raut, is pursuing his Ph.D. degree in Computer Science. He has completed his M.Sc. degree in Computer Science from University Department of Computer Science and Information Technology from Dr.B.A.M.U. Aurangabad and is working as the Assistant Professor at Department of Computer Science and Application, School of Computational Sciences, Solapur University, Solapur. He is Member of IACSIT Singapore, Member of IAEng, Hong Kong, and CSTA, New York. He worked as the Research Scholar and submitted a project in Computer Science under the UGC SAP (II) DRS Phase-I: 2009-2014 under the theme "Biometric: Multimodal System Development”. 